\title{
A Hilbert Type Inequality for Finite Series
}

\section{Baoju Sun}

Department of Mathematics

Zhejiang University of Water Resources and Electric Power, Hangzhou, Zhejiang 310018, China sunbj@zjweu.edu.cn

Keywords: Hilbert Type inequality; Cauchy inequality; monotonicity of functions

Abstract. In this paper, by using Cauchy inequality, monotonicity of functions, Hilbert type inequality with finite series version is established.

\section{Introduction}

If $a_{n} \geq 0, b_{n} \geq 0, m, n \in \mathrm{N}$, such that $0<\sum_{n=1}^{\infty} a_{n}^{2}<\infty, 0<\sum_{n=1}^{\infty} b_{n}^{2}<\infty$, then the well known Hilbert inequalities are given by

$$
\begin{aligned}
& \sum_{n=1}^{\infty} \sum_{m=1}^{\infty} \frac{a_{m} b_{n}}{m+n}<\pi\left(\sum_{n=1}^{\infty} a_{n}^{2}\right)^{1 / 2}\left(\sum_{n=1}^{\infty} b_{n}^{2}\right)^{1 / 2} . \\
& \sum_{n=1}^{N} \sum_{m=1}^{N} \frac{a_{m} b_{n}}{m+n}<\pi\left(\sum_{n=1}^{N} a_{n}^{2}\right)^{1 / 2}\left(\sum_{n=1}^{N} b_{n}^{2}\right)^{1 / 2} .
\end{aligned}
$$

Hilbert type inequalities are given by

$$
\begin{gathered}
\sum_{n=1}^{\infty} \sum_{m=1}^{\infty} \frac{a_{m} b_{n}}{\max (m, n)}<4\left(\sum_{n=1}^{\infty} a_{n}^{2}\right)^{1 / 2}\left(\sum_{n=1}^{\infty} b_{n}^{2}\right)^{1 / 2} . \\
\sum_{n=1}^{N} \sum_{m=1}^{N} \frac{a_{m} b_{n}}{\max (m, n)}<4\left(\sum_{n=1}^{N} a_{n}^{2}\right)^{1 / 2}\left(\sum_{n=1}^{N} b_{n}^{2}\right)^{1 / 2} . \\
\sum_{n=1}^{\infty} \sum_{m=1}^{\infty} \frac{|\ln (m / n)| a_{m} b_{n}}{\max (m, n)}<8\left(\sum_{n=1}^{\infty} a_{n}^{2}\right)^{1 / 2}\left(\sum_{n=1}^{\infty} b_{n}^{2}\right)^{1 / 2} . \\
\sum_{n=1}^{N} \sum_{m=1}^{N} \frac{|\ln (m / n)| a_{m} b_{n}}{\max (m, n)}<8\left(\sum_{n=1}^{N} a_{n}^{2}\right)^{1 / 2}\left(\sum_{n=1}^{N} b_{n}^{2}\right)^{1 / 2} .
\end{gathered}
$$

(see Hardy et al.[1]). In recently years, various improvements and extensions of the Hilbert inequality and Hilbert type inequalities appear in a great deal of papers (see [2-5]). Zhang xiaoming, Chu yuming ([2]) gave improvement of (2) as:

$$
\pi^{2} \sum_{n=1}^{N} a_{n}^{2} \sum_{n=1}^{N} b_{n}^{2}-\left(\sum_{n=1}^{N} \sum_{m=1}^{N} \frac{a_{m} b_{n}}{m+n}\right)^{2} \geq \min _{1 \leq n \leq N}\left\{n a_{n}^{2}\right\} \min _{1 \leq n \leq N}\left\{n b_{n}^{2}\right\}\left[\pi^{2}\left(\sum_{n=1}^{N} \frac{1}{n}\right)^{2}-\left(\sum_{n=1}^{N} \sum_{m=1}^{N} \frac{1}{\sqrt{m n}(m+n)}\right)^{2}\right]
$$

The major objective of this paper is to formulate new inequalities, which is improvement of (6).

\section{Some lemmas}

In order to prove our main result we need some lemmas, which we present in this section.

Lemma 1 If $1 \leq n \leq N, n, N$ are positive integers, then 


$$
\begin{gathered}
\sum_{m=1}^{N} \frac{|\ln (m / n)|}{\sqrt{m} \cdot \max (m, n)}<8 n^{-1 / 2} . \\
\sum_{m=1}^{N+1} \frac{\mid \ln (m /(N+1) \mid}{\sqrt{m} \cdot \max (m, N+1)}<4(N+1)^{-1 / 2} .
\end{gathered}
$$

( iii )

$$
\sum_{m=1}^{N} \sum_{n=1}^{N} \frac{|\ln (m / n)|}{\sqrt{m n} \cdot \max (m, n)}<8 \sum_{n=1}^{N} \frac{1}{n}
$$

Proof. ( i ) see[7].

(ii )

$\sum_{m=1}^{N+1} \frac{|\ln (m /(N+1))|}{\sqrt{m} \cdot \max (m, N+1)}<\int_{0}^{N+1} \frac{|\ln (x /(N+1))|}{(N+1) \sqrt{x}} d x=\int_{0}^{1} \frac{-\ln t}{\sqrt{N+1} \sqrt{t}} d t=4(N+1)^{-1 / 2}$.

( iii )

$$
\sum_{m=1}^{N} \sum_{n=1}^{N} \frac{|\ln (m / n)|}{\sqrt{m n} \cdot \max (m, n)}<\sum_{n=1}^{N} \frac{1}{\sqrt{n}} 8 n^{-\frac{1}{2}}=8 \sum_{n=1}^{N} \frac{1}{n} \text {. }
$$

Lemma 2 Let $f(N)=64\left(\sum_{n=1}^{N} \frac{1}{n}\right)^{2}-\left(\sum_{m=1}^{N} \sum_{n=1}^{N} \frac{|\ln (m / n)|}{\sqrt{m n} \cdot \max (m, n)}\right)^{2}$, then $f(N+1)>f(N)$.

Proof. $f(N+1)-f(N)$

$$
\begin{aligned}
& =64\left(2 \sum_{n=1}^{N} \frac{1}{n}+\frac{1}{N+1}\right)-\left(\frac{2}{\sqrt{N+1}} \sum_{n=1}^{N+1} \frac{|\ln (n /(N+1))|}{\sqrt{n} \cdot \max (N+1, n)}\right) \times\left[2 \sum_{n=1}^{N} \sum_{m=1}^{N} \frac{|\ln (m / n)|}{\sqrt{m n} \cdot \max (m, n)}\right. \\
& \left.+\frac{2}{\sqrt{N+1}} \sum_{n=1}^{N+1} \frac{|\ln (n /(N+1))|}{\sqrt{n} \cdot \max (N+1, n)}\right] \\
& >64\left(2 \sum_{n=1}^{N} \frac{1}{n}+\frac{1}{N+1}\right)-\frac{8}{N+1}\left(16 \sum_{n=1}^{N} \frac{1}{n}+\frac{8}{N+1}\right)=0 .
\end{aligned}
$$

\section{Main results}

Theorem 1 If $a_{n} \geq 0, b_{n} \geq 0, n=1,2, \mathrm{~L}, N . \quad N$ is positive integer, then

$$
\begin{aligned}
& 64 \sum_{n=1}^{N} a_{n}^{2} \sum_{n=1}^{N} b_{n}^{2}-\left(\sum_{n=1}^{N} \sum_{m=1}^{N} \frac{|\ln (m / n)| a_{m} b_{n}}{\max (m, n)}\right)^{2} \\
& \geq \min _{1 \leq n \leq N}\left\{n a_{n}^{2}\right\} \min _{1 \leq n \leq N}\left\{n b_{n}^{2}\right\}\left[64\left(\sum_{n=1}^{N} \frac{1}{n}\right)^{2}-\left(\sum_{n=1}^{N} \sum_{m=1}^{N} \frac{|\ln (m / n)|}{\sqrt{m n} \cdot \max (m, n)}\right)^{2}\right]
\end{aligned}
$$

Proof. Let $c_{n}=\sqrt{n} a_{n}, d_{n}=\sqrt{n} b_{n}$, then inequality (11) is translated into 


$$
\begin{aligned}
& 64 \sum_{n=1}^{N} \frac{c_{n}^{2}}{n} \sum_{n=1}^{N} \frac{d_{n}^{2}}{n}-\left(\sum_{n=1}^{N} \sum_{m=1}^{N} \frac{|\ln (m / n)| c_{m} d_{n}}{\sqrt{m n} \cdot \max (m, n)}\right)^{2} \\
\geq & \min _{1 \leq n \leq N}\left\{c_{n}^{2}\right\} \min _{1 \leq n \leq N}\left\{d_{n}^{2}\right\}\left[64\left(\sum_{n=1}^{N} \frac{1}{n}\right)^{2}-\left(\sum_{n=1}^{N} \sum_{m=1}^{N} \frac{|\ln (m / n)|}{\sqrt{m n} \cdot \max (m, n)}\right)^{2}\right] .
\end{aligned}
$$

By using Cauchy inequality, we have

$$
\begin{aligned}
& 64 \sum_{n=1}^{N} \frac{c_{n}^{2}}{n} \sum_{n=1}^{N} \frac{d_{n}^{2}}{n}-\left(\sum_{n=1}^{N} \sum_{m=1}^{N} \frac{|\ln (m / n)| c_{m} d_{n}}{\sqrt{m n} \cdot \max (m, n)}\right)^{2} \\
\geq & 64 \sum_{n=1}^{N} \frac{c_{n}^{2}}{n} \sum_{n=1}^{N} \frac{d_{n}^{2}}{n}-\sum_{n=1}^{N} \sum_{m=1}^{N} \frac{|\ln (m / n)| c_{m}^{2}}{\sqrt{m n} \cdot \max (m, n)} \sum_{n=1}^{N} \sum_{m=1}^{N} \frac{|\ln (m / n)| d_{n}^{2}}{\sqrt{m n} \cdot \max (m, n)} .
\end{aligned}
$$

Let $f\left(c_{1}, c_{2}, \mathrm{~L}, c_{n}, d_{1}, d_{2}, \mathrm{~L}, d_{n}\right)$

$$
=64 \sum_{n=1}^{N} \frac{c_{n}^{2}}{n} \sum_{n=1}^{N} \frac{d_{n}^{2}}{n}-\sum_{n=1}^{N} \sum_{m=1}^{N} \frac{|\ln (m / n)| c_{m}^{2}}{\sqrt{m n} \cdot \max (m, n)} \sum_{n=1}^{N} \sum_{m=1}^{N} \frac{|\ln (m / n)| d_{n}^{2}}{\sqrt{m n} \cdot \max (m, n)} .
$$

to compute the partial derivatives of a function $f\left(c_{1}, c_{2}, \mathrm{~L}, c_{n}, d_{1}, d_{2}, \mathrm{~L}, d_{n}\right)$ with respect to $c_{i} . d_{i}$, and using lemma 1 , we have

$$
\begin{aligned}
& \frac{\partial f}{\partial c_{l}}=64 \cdot \frac{2 c_{i}}{i} \sum_{n=1}^{N} \frac{d_{n}^{2}}{n}-\sum_{n=1}^{N} \frac{2 c_{i}|\ln (i / n)|}{\sqrt{i} \sqrt{n} \cdot \max (i, n)} \sum_{n=1}^{N} \frac{d_{n}^{2}}{\sqrt{n}}\left(\sum_{m=1}^{N} \frac{|\ln (m / n)|}{\sqrt{m} \cdot \max (m, n)}\right) \\
& >\frac{128 c_{i}}{i} \sum_{n=1}^{N} \frac{d_{n}^{2}}{n}-\frac{2 c_{i}}{\sqrt{i}} \cdot 8 i^{-1 / 2} \sum_{n=1}^{N} \frac{d_{n}^{2}}{\sqrt{n}} 8 n^{-1 / 2}=0 .
\end{aligned}
$$

thus $f\left(c_{1}, c_{2}, \mathrm{~L}, c_{n}, d_{1}, d_{2}, \mathrm{~L}, d_{n}\right)$ is monotone increasing for $c_{i}$. In a similar way we can provde that $\frac{\partial f}{\partial d_{i}}>0$, and this implies $f\left(c_{1}, c_{2}, \mathrm{~L}, c_{n}, d_{1}, d_{2}, \mathrm{~L}, d_{n}\right)$ is monotone increasing for $d_{i}$. We obtain $f\left(c_{1}, c_{2}, \mathrm{~L}, c_{n}, d_{1}, d_{2}, \mathrm{~L}, d_{n}\right)$

$\geq f\left(\min _{1 \leq n \leq N}\left\{c_{n}\right\}, \min _{1 \leq n \leq N}\left\{c_{n}\right\}, \mathrm{L}, \min _{1 \leq n \leq N}\left\{c_{n}\right\}, \min _{1 \leq n \leq N}\left\{d_{n}\right\}, \min _{1 \leq n \leq N}\left\{d_{n}\right\}, \mathrm{L}, \min _{1 \leq n \leq N}\left\{d_{n}\right\}\right)$.

In view of (13), (12) holds. The theorem is proved.

From theorem 1 and lammas 2, we have

Theorem 2 If $a_{n} \geq 0, b_{n} \geq 0, n=1,2, \mathrm{~L}, N . \quad N$ is positive integer, then

$$
64 \sum_{n=1}^{N} a_{n}^{2} \sum_{n=1}^{N} b_{n}^{2}-\left(\sum_{n=1}^{N} \sum_{m=1}^{N} \frac{|\ln (m / n)| a_{m} b_{n}}{\max (m, n)}\right)^{2} \geq 64 \min _{1 \leq n \leq N}\left\{n a_{n}^{2}\right\} \min _{1 \leq n \leq N}\left\{n b_{n}^{2}\right\} .
$$

\section{References}

[1] G. H. Hardy, J. E. Littlewood and G. Pólya, Inequalities, Gambridge University Press, Gambridge, 1952.

[2] X. Zhang, Y. Chu, New discussion to analytic inequalities, Harbin institute of technology press, 2009.

[3] M. Gao, B. Yang, On the extended Hilbert's inequality, Proceeding of the American Mathematical Society 126 (1998), no.3, 751-759

[4] J. Kuang, L. Debnath, On new generalizations of Hilbert's inequality and their applications, Journal of Mathematical Analysis and Applications 245 (2000), no.1, 248-265. 
[5] B. Yang, On a strengthened version of the more accurate Hardy-Hilbert's inequality, Acta Mathematica, Sinica (China) 42 (1999), no.6, 1103-1110.

[6] B. Yang, On a Base Hilber-type inequality, Journal of Guangdong Education Institute(Science Edition), 26(2006), no.3, 1-5.

[7] B. Yang, A Hilber-type inequality with two pairs of conjugate exponents, Journal of JiLin University (Science Edition),45(2007), no.4, 524-528.

[8] B. Sun. A Multiple Hilbert type integral inequality with the best contant factor [J]. Journal of Inequalities and Applications, 2007. 